\title{
TRA LETTERATURA E VERITÀ: IMMAGINARIO LANDOLFIANO NEL «RACCONTO D'AUTUNNO»
}

\author{
Eva Mesárová \\ Università di Matej Bel, Banská Bystrica (Slovacchia) \\ eva.mesarova@umb.sk \\ «Il più bel romanzo sulla Resistenza e uno \\ dei più belli in assoluto è Racconto d'autunno» \\ (Giovanni Ungarelli, dirigente della casa editrice Rizzoli)
}

\section{Riassunto}

Tommaso Landolfi (1908-1979), è un autore appartenente alla cultura italiana e al tempo stesso a quella europea. Nel 1947 scrive il breve romanzo Racconto d'autunno. L'opera rievoca la distruzione, da parte delle artiglierie americane, della millenaria Abbazia di Montecassino e dei luoghi più cari della sua infanzia, che l'autore visse (espressione che ricorre, appunto, in quest'opera) come una vera e propria profanazione. Si tratta di un romanzo ricco di significati metaforici, a cominciare dalla guerra, metafora della crudele insensatezza degli uomini, fino alla casa stessa, che il protagonista incontra sulla sua strada. Alla luce di certi dati biografici, il presente contributo si propone di analizzare questa dimensione tragica e anche mitica del dolore, che Landolfi prova di fronte alla casa violata dagli uomini e dalla devastazione causata dagli uomini in fuga. La guerra aveva, infatti, profanato la nobile dimora di Pico, luogo che aveva assistito alla stesura di tutte le opere della sua prima stagione ed era per lui una sorta di guscio protettivo. Lo studio si sofferma in particolare sulla ricezione di alcune esperienze belliche (i bombardamenti e gli altri eventi bellici avvenuti in Ciociaria nel 1943) nella cultura italiana del tempo.

Parole chiave: cultura italiana, Landolfi, immaginario, casa 
Fra i motivi che m'inducono a presentare lo studio su un libro di Tommaso Landolfi (1908 - 1979), spicca soprattutto la volontà di mettere in luce uno scrittore molto ammirato, come testimoniano i numerosi premi letterari da lui ottenuti, ma poco letto dal pubblico italiano e internazionale, nell'anno del centodecimo anniversario della sua nascita.

Negli anni Cinquanta si aprivano all'Università di Roma, in margine all'insegnamento di Storia della Letteratura italiana (la cui cattedra ricoprì Natalino Sapegno) dibattiti di contemporaneistica. Un tema favorito, ricorda Siciliano «era la narrativa della resistenza. Che la guerra civile, avvenuta poco più di dieci anni prima, potesse essere raccontata in modo diverso dal corrivo naturalismo di Renata Viganò ${ }^{[1]}$, e in modo ancora più diverso dall'imperiosa allusività di Uomini e $n o^{[2]}, \mathrm{mi}$ sembrò un miracolo» (Siciliano, 2009, 267).

Con il romanzo Racconto d'autunno, pubblicato nel 1947 presso Vallecchi, ci troviamo di fronte a un'opera fantastica, tipicamente landolfiana e profondamente autobiografica. Nel gennaio del 1944 a Pico (provincia di Frosinone - Ciociaria) i tedeschi ordinano lo sgombero della popolazione che andrà a Priverno, a Roma e in centri del Nord. Per dieci giorni, dal 12 al 22 di maggio del 1944, Pico subisce un duro bombardamento: la casa di Landolfi, il luogo prediletto dell'infanzia e della fanciullezza, in cui scrive quasi tutte le sue opere, ne esce gravemente danneggiata ed è per qualche tempo ricetto per le numerose famiglie di sfollati che ne completano la devastazione. Dalla fine di ottobre 1943 alla fine di maggio 1944, lo stesso palazzo Landolfi in paese è occupato (Bo, 1991, XLIX-L):

Prima tedeschi, quindi francesi e truppe alleate ne fanno il loro quartier generale, depredandolo, al momento di andar via, della mobilia antica e degli arredi di pregio (di cui era, come ogni residenza signorile, ampiamente dotato); nonché, da parte dei colti ufficiali, dei libri più preziosi tra quelli della vastissima biblioteca: ed è per tal motivo, appunto, che è assai difficile ricostruire in maniera esauriente il genere di testi frequentati da Landolfi fino a quel momento; anche se la sfaccettata

[1] Renata Viganò (1900 - 1976), una scrittrice e partigiana italiana, a soli 12 anni riuscì a far pubblicare, nel 1912, la sua prima raccolta di poesie, Ginestra in fiore, seguita nel 1915 da Piccola Fiamma, ma raggiunse una certa notorietà solamente nel 1949 con L'Agnese va a morire, romanzo d'impianto neorealistico tra i più intensi della narrativa ispirata alla Resistenza.

[2] Uomini e no è un romanzo di Elio Vittorini, scritto tra la primavera e l'autunno del 1944, ma pubblicato solo nel giugno del 1945. Questo è probabilmente il primo romanzo della Resistenza. 
e profonda cultura ch'egli in ogni occasione dimostrava, presuppone la lettura del maggior numero possibile di autori di ogni letteratura.

Il breve romanzo Racconto d'autunno, narrato in prima persona, è ambientato nell'Italia della Seconda guerra mondiale. Landolfi lo scrive tra il settembre e l'ottobre del 1946 a Firenze «tranne un qualche periodo al Forte e poi a Pico (dove intanto sono intrapresi i più urgenti lavori di ricostruzione) tra l'estate e l'autunno, sotto la viva impressione degli eventi bellici» (Landolfi, 1991, 51).

Veniamo alla vicenda narrata. Il punto di partenza del libro è dato dalla fuga del protagonista - narratore, un partigiano sbandato (Landolfi, 1991, 437):

La guerra m'aveva sospinto all'epoca di questa storia, lontano dai miei abituali luoghi di residenza. Due formidabili eserciti stranieri si scontravano allora sul nostro suolo, conducendo una campagna cruenta e che parve infinita alla maggior parte della popolazione, la quale ne fu, come si immagina, direttamente e barbaramente danneggiata.

La resistenza ci appare, nel primo capitolo, come il gioco del nascondino (Landolfi, 1991, 437):

Inoltre le esose pretese, in uomini e materiali, d'uno di questi eserciti (l'invasore, che lentamente s'andava ritirando, attraverso il paese, davanti all'altro, detto liberatore), nonché spirito patriottico o compromissione politica, costrinsero numerosissime persone a cercar rifugio per lunghi mesi o anche per anni in posti selvaggi e discosti dalle grandi strade, abbandonando i propri interessi, i propri averi e le famiglie medesime.

I due eserciti, uno l'«invasore» e l'altro «detto liberatore», sono il riferimento a una categorizzazione più ampia tramite la quale si cerca di aderire alla realtà che si ha di fronte e che serve come materia della storia. Ciò che lo scrittore offre al lettore è lo spirito del tempo, la tendenza culturale predominante in una determinata epoca: «Dove, coloro che ne avevano la possibilità o se ne sentirono il genio, si organizzarono per una resistenza armata $\mathrm{o}$ addirittura per l'offesa, altri resisterono almeno passivamente alle imposizioni degli invasori, altri infine badarono a togliersi dal folto della mischia» (Landolfi, 1991, 437). 
Un aspetto morale e politico diventano presto un sentire del tempo, un sentimento sparso nell'aria e del quale ciascuno fa quello che vuole e quello che sa. Landolfi così scrive di un fatto di memoria collettiva (Landolfi, 1991, 437):

Perché, dico, appartenevo a una di queste categorie, la mia vita fu lungamente quella del bandito, anzi, avuto riguardo ai luoghi più o meno impervi che frequentavo, del brigante, di continuo braccato. Molti divisero meco tale vita, e da essi e ad essi fui a volta dalle circostanze separato e riunito.

Stanco e affamato, il protagonista si aggira nella boscaglia sotto una pioggia insistente, nel tentativo di eludere le attenzioni delle pattuglie di un esercito invasore. Durante un'ennesima fuga, scopre in mezzo alle montagne una strana e antica dimora, un rifugio che lo protegge dalle violenze della guerra (Landolfi, 1991, 439-440):

Quanto cercavo con poca speranza era lì ai miei piedi, a qualche tiro di fucile: una casa! Dal cui camino, per giunta, si elevava, nell'aria umida e quasi fosca, una fumata scompigliata dal vento, assai malinconica forse di per se stessa, ma che a me apparve non so dir quanto allegra. Il cuore mi si aprì, mi mossi verso la provvidenziale dimora.

La casa offre, in quel momento, un rifugio per ripararsi, una postazione da cui potenzialmente resistere a un attacco e quindi rappresenta una posizione da conquistare (Landolfi, 1991, 440):

Essa era posta su una specie di minuscolo altipiano, sto per dire ballatoio, d'ogni parte circondato da groppe o colli, che la montagna formava in quel luogo, ove confluivano due o tre massicce pendici. Circondata da alcune dipendenze, appariva grande e di aspetto dignitoso, una residenza signorile o un maniero, piuttosto che una fattoria.

Vengono descritti la casa, che ha un aspetto abbandonato e i tentativi del protagonista di penetrarvi. Scopre, poi, che ci abita uno strano vecchio, ombroso, ma di modi e d'aspetto nobili, il quale anche se infastidito dalla sua presenza, lo ospiterà.

La situazione cambia, il maniero si rivela un vero e proprio labirinto 
(le numerose stanze che compongono la casa si articolano e sboccano le une dalle altre), «trasparente controfigura della casa avita, l'avo materno ${ }^{[3]}$ di Pico Farnese, nonché immagine anche troppo fedele della casa Usher di Poe e del castello dei Carpazi di Verne» (Amigoni, 2004, 74). La dimora stregata si rivela essere una sorta di labirinto di Creta, ma a differenza di quanto accade a Teseo nel mito greco, la ricerca del protagonista non è diretta verso l'uscita del labirinto, bensì verso il suo centro, che lo attrae magneticamente (Durand, 2009). Al centro della casa-labirinto non si trova il Minotauro, ma un'altra creatura mostruosa, una donna affascinante, l'immagine di un antico ritratto della giovane moglie morta, Lucia. Il protagonista scopre nel labirintico maniero una specie di sotterraneo nel quale assiste agli strani riti evocatori compiuti dal vecchio - si forma una strana larva, che ha l'aspetto della donna del ritratto, ma al protagonista appare terribile e cupa. ${ }^{[4]}$

Il vecchio pazzo e il narratore vedono lo stesso mostro, il vecchio si sente scoperto e minaccia l'uomo, il quale si dà alla fuga. Dopo una serie di avventure torna nella casa e incontra una strana fanciulla, Lucia, somigliante alla donna del quadro, la quale gli rivela tutta una serie di cose. La morte del padre (il vecchio nobile), la propria lunga prigionia in quella stanza buia, dove il padre la tratteneva gelosamente, avendo riversato su di lei, dopo la morte della moglie, l'amore fortissimo che aveva nutrito per la defunta. La figlia aveva assistito fin da piccola agli strani riti sado - masochistici tra il padre e la madre e in seguito i genitori li avevano compiuti anche su di lei. Il protagonista prova per lei un sentimento misto, l'amore e la tenerezza si alternano alla pietà (la fanciulla soffre anche di epilessia) e alla repulsione.

Il romanzo si conclude con l'arrivo di alcuni soldati e con la morte della fanciulla per un colpo di pistola. A un anno di distanza, quando la casa è ormai sventrata, il protagonista visita la tomba di Lucia (Landolfi, 1991, 515):

[3] Cfr. Tommaso Landolfi, Un amore del nostro tempo, 1965, in Opere II (1960-1971), a cura di Idolina Landolfi, Milano, Rizzoli, 1992, p. 557.

[4] «Vedevo, ecco... Ma che cosa chi vedevo? Certo non era che un'illusione dei miei sensi. E di nuovo ella (chi altri? perché ho detto or ora creatura sconosciuta?), ella con disperata violenza, con cieca disperazione, con oscura protervia, voleva in quel fumo incarnarsi, e di nuovo qualcosa o qualcuno glielo impediva, la respingeva. Ma ella era ormai qui. No, che ahimè non v'era, non v'era più da gran tempo, e mai più vi sarebbe stata! Eppure io sentivo... Che cosa potevo io sentire, nel mio stato? Erano i miei nervi scossi e nulla più». (Landolfi, 1991, 491). 
Sulla sua tomba, il cespo di roselline d'autunno da me piantato è divenuto grande e forte, e ogni anno, fra gli altri fiori, ne dà uno particolarmente bello. Guardandolo, ricordo sempre le parole sue, o della sua "voce": «di dove si sta facendo tanto bella quella rosellina, se non col tuo sangue?». Ora è davvero così.

Landolfi era solito ritirarsi nel paese natale, nella casa avita che è la protagonista di tanti suoi racconti, e dove soprattutto egli lavorò anche dopo il 1956, quando si sposò e si stabilì a San Remo (Landolfi, 1998, 117):

Punto primo: la penna che laggiù [a Pico] correva qui [a San Remo] s'impunta e per avviarla ci vuol la mano di Dio. Non è un'immagine, parlo della penna in carne e ossa; anche l'anno passato, qui, faceva il medesimo lavoro, e tornata laggiù riprese a correre. A che si debba il fatto, se all'inchiostro, all'aria del luogo o a più seri e segreti motivi, non so.

Landolfi scrive, dunque, il breve romanzo Racconto d'autunno a Pico, nel palazzo devastato dai bombardamenti, quando era ancora fresco il ricordo della scellerata distruzione della millenaria Abbazia di Montecassino (15 febbraio 1944) e dei luoghi più cari della sua infanzia, che egli visse (è un'espressione che ricorre, appunto, in quest'opera) come una vera e propria profanazione. Nel quarto capitolo il narratore ci rivela la situazione attraverso la voce del vecchio nobile (Landolfi, 1991, 452-453):

Raddoppiai di zelo e fui infine premiato dalla seguente frase, che non era già una risposta alle mie domande, ma come alla situazione in generale: "Qui non entra mai nessuno». Seguita subito dall'altra, arrossendo a un tratto il vecchio di collera: «O meglio, non era mai, ma costoro 1'hanno profanata». Tradussi mentalmente, frammezzo alla profonda storditezza e noncuranza che mi tenevano: «o meglio, qui non era mai entrato nessuno, ora i soldati di quest'esercito hanno profanato la casa»; né mi soffermai a giudicare quanto poteva esservi d'orgoglioso o doloroso nel definire profanazione una violazione di domicilio».

L'abbazia di Montecassino si trova solo a pochi chilometri dalla dimora dei suoi avi. È stata, purtroppo, solo l'ignoranza e la superficialità di un generale alleato che condannò a un'inutile distruzione l'Abbazia, da parte delle artiglierie americane. 
Landolfi prova dolore di fronte alla casa, violata. Nel maggio 1944, durante il passaggio del fronte, Tommaso Landolfi si trova a Roma (con suo padre) e ritornano tutti e due a Pico alla fine di luglio. Lo spettacolo che si offre ai loro occhi è scoraggiante (Landolfi, 1991, 762):

Di qui, da questa casa, è passata la guerra, lasciandovi vaste piaghe aperte, lasciandovi in ispecie, nell'aria, le tracce della sua insolenza. Quando vi tornammo, poco dopo, non solo due quartieri erano crollati, e le mura, un tempo così gelose del loro vetusto segreto, si scosciavano al sole, ma, quel che era peggio, esse avevano dato ricetto a molta gente straniera, a Tedeschi prima, poi a Francesi o Algerini, infine a torme di 'sfollati'. Noi contemplavamo quello squallore e quella rovina, ma il peggio come ho detto, era che non riconoscevamo l'aria di casa nostra.

La rovina della dimora lascia una traccia permanente nell'animo dello scrittore anche molti anni dopo, nel 1968 (Landolfi, 1968):

La casa medesima non è più quella, dopo il passaggio della guerra e coi barbari di recentissimo conio che presentemente vi spadroneggiano. Né intendo delle sue materiali devastazioni, ma dell'aura segreta ed inebriante un tempo per tutto diffusa, oggi svaporata, o rifugiata in certo stipo a muro: quasi minuscolo tempio ad altri dèi, colmo d'oggettucci inutili e tragici nella loro impotenza, nel loro ostinato, antico sentore.

Tedeschi, plebe, ladri, / Tempo, morte, corruzione, / Sono i flagelli caduti / Sul Ricettacolo dei sogni; / E cieche strida di femmine / E vile pianto di bambini. / E ciò ch'era geloso e segreto / Si fece pubblico ed impronto; / E degli antichi corpi rudi / I sacconi persero l'impronta, / Né i nuovi, delicati e dispettosi, / Seppero alcuna segnalarne; / E l'odore dei pannamenti appesi / Nei crepitanti armadi, a poco a poco / Svanì nell'aria (Landolfi, 1977, 49).

Nel capitolo diciottesimo troviamo di nuovo i motivi dell'offesa e della difesa, della resistenza e della lotta, quando il narratore e Lucia, vengono aggrediti da dei banditi. Giungono alcuni soldati «bruni di carnagione e d'uniforme»: si tratta di uomini regolari appartenenti a quei reparti di truppe coloniali, messi in campo dall'esercito anglo-americano (che Landolfi con sottile ironia chiama: «esercito liberatore») durante il secondo conflitto mondiale, i quali si scagliano contro la fanciulla per seviziarla e poi senza scrupoli le sparano due colpi di pistola. 
Il narratore non solo scorge all'interno degli eventi storici un filo immaginario, ma nel romanzo esprime un giudizio negativo su una delle pagine più mostruose vissute dalla popolazione italiana civile durante la Seconda guerra mondiale: la verità nascosta delle «marocchinate», saccheggi e stupri delle truppe francesi in mezza Italia (Landolfi, 1991, 511):

[...] vi sopravvenivano tre soldati d'una razza e d'un corpo a me sconosciuti; quattro o cinque altri ne sbucarono di lì a un istante da ambo i lati della casa, che avevano aggirata. Bruni di carnagione e d'uniforme, parendo alle labbra e agli occhi gente d'Africa, avevano lunghi capelli inanellati sotto l'elmetto e cerchi d'oro alle orecchie; un paio di loro tiravano per la cavezza due muletti carichi di munizioni e provviste. È inutile soggiunga che nulla di buono presagivano i loro ceffi, i quali avevano alcunché di crudele, di belluino e persin di diabolico.

[...] costoro appartenevano a quei reparti di truppe coloniali che l'esercito liberatore aveva messi in campo onde far impeto contro posizioni montane giudicate altrimenti inespugnabili; il qual compito, a vero dire, assolsero egregiamente, poiché erano adusati a un tal genere di guerra. Ma essi, che in tempi precedenti avevano avuto a subire gravi torti, nel loro paese medesimo, dai nostri connazionali, giungevano ora qui colla sete della vendetta e l'animo dei saccheggiatori e degli stupratori, né, ebbri di conquista, si brigavano di distinzioni purchessia fra amici e nemici, armati e non.

Lo scrittore li chiama anche «banda di depredatori che si dilungavano, non poco spesso, dai loro quartieri» perché «cercassero preda, vino, e soprattutto donne» (Landolfi, 1991, 512). Scrive Verdenelli a proposito della scrittura landolfiana: «Landolfi parte sempre dal reale per arrivare a un certo risvolto surreale, registrando in maniera quasi cronachistica un evento particolarmente crudo e violento» (Verdenelli, 1997, 178).

Con il termine «marocchinate» vengono generalmente definiti gli stupri di gruppo, le uccisioni, i saccheggi e le violenze di ogni genere perpetrate dalle truppe coloniali francesi (goumier francesi inquadrati nel CEF - Corpo di spedizione francese in Italia (Corps expéditionnaire français en Italie), aggregate agli Alleati, ai danni della popolazione italiana, dei prigionieri di guerra e perfino di alcuni partigiani comunisti durante la campagna d'Italia della Seconda guerra mondiale. 
Il 14 maggio 1944 i goumier del Corpo di spedizione francese in Italia, attraversando un terreno apparentemente insuperabile sui monti Aurunci, aggirarono le linee difensive tedesche nell'adiacente Valle del Liri, consentendo al XIII Corpo britannico di sfondare la linea Gustav e di avanzare fino alla successiva linea di difesa predisposta dalle truppe germaniche, la linea Adolf Hitler (Strazza, 2010, 110-111). A Pico i soldati statunitensi del $351^{\circ}$ reggimento fanteria (della $88^{a}$ divisione di fanteria), i cui membri erano soprannominati $\mathrm{i}$ «blue devils» per la loro ferocia in combattimento, giunsero mentre i goumier stavano compiendo le violenze, ma furono bloccati dal comandante francese del reparto, che disse loro che «erano qui per combattere i tedeschi e non i francesi» (Strazza, 2010, 110-111). Poi, in una relazione redatta il 28 maggio 1944 del capitano italiano Umberto Pittali, viene detto che gli «ufficiali francesi lasciano ai marocchini una discreta libertà di azione»e «preferiscono ignorare» (Strazza, 2010, 111) quanto accade. ${ }^{[5]}$

Il romanzo è ispirato a fatti storici riversati nelle esperienze individuali finzionali del protagonista. Anche nella parte conclusiva del romanzo sarà ripreso il tema della guerra e in un quadro di desolanti rovine, se ne registrerà la fine: «La guerra era ormai passata di là, la vera e grande guerra, con tutto il suo fragore e le sue rovine, ma s'era già dileguata lontano; ne rimanevano le tracce desolate» (Landolfi, 1991, 514). È la stessa casa di Racconto d'autunno, su cui la guerra ha infierito:

Quel luogo aveva dovuto divenire per un tempo posizione difensiva e la casa fortilizio, che i colpi avversi non avevano risparmiato. Essa giaceva sventrata, mostrando le sue viscere, sorpresa dalla luce nei suoi più intimi segreti, nei suoi cunicoli, nei suoi passaggi un tempo nascosti entro lo spessore delle vecchie muraglie, in quanto rimaneva delle sue suppellettili, gelosamente sacre un tempo alcune, delle sue tappezzerie che ora pendevano come lembi di carne disseccata: lamentevolmente vuota del suo mistero, che era come il suo sangue; trapassata dal cielo (Landolfi, 1991, 514).

Quanto al genere, Edoardo Sanguineti osserva che si tratta di una «scommessa arrischiata», di «una specie di narrativa del mistero, tra il

[5] Da documenti dell'Archivio Centrale dello Stato risulta che anche i francesi bianchi parteciparono alle violenze: a Pico furono, infatti, violentate 51 donne (di cui nove minorenni) da 181 franco-africani e da 45 francesi bianchi. Dato questo episodio e considerando che i francesi europei costituivano il $40 \%$ di tutto il Cef, risulta limitativo addossare la responsabilità delle violenze ai soli goumiers marocchini. 
poliziesco e il liberty» e aggiunge: «chi ci capisce è bravo» (Sanguineti, 2010, 160). I critici ascrivono il romanzo di cui ci stiamo occupando per lo più al fantastico; Ferdinando Amigoni definisce l'opera "perfettamente fantastica» (Amigoni, 2004, 74). Il fantastico, come è ben noto, introduce in un avvenimento reale uno inverosimile, che esula dalle norme da tutti accettate. Nelle sue opere, Landolfi gioca ripetutamente con spettri e altri esseri sovrannaturali cari alla narrativa fantastica dell'Ottocento e Racconto d'autunno non ne è che un esempio. Lo scrittore non tenta di spiegare il fantasma, lo «spirito d'inferno, una immonda larva» (Landolfi, 1991, 492), che ha l'aspetto della donna del ritratto, donna di fumo che torna dal regno dei morti, secondo un rito magico. «Non spero di farmi intendere, forse non voglio. Non pretendo giustificarmi né dare spiegazioni; solo dirò quello che vidi» (Landolfi, 1991, 491). Il romanzo, però, non si chiude ponendo l'attenzione sul fantasma e l'epilogo, non a caso, non è fantastico. Ancora una volta «l'impossibilità di convivenza tra vivi e spiriti, vista in termini letterari, corrisponde all'impossibilità da parte del modo fantastico di trovare un suo spazio nella letteratura contemporanea» (Zangrandi, 2011, 190).

Landolfi, per il titolo del suo penultimo lavoro Se non la realtà, si è ispirato alla proposizione con cui si conclude il Commiato di Ombre: «Non v'è più meta alle nostre pigre passeggiate, se non la realtà» (Landolfi, 1954, 183). Va peraltro notato che nelle sue opere non ha mai smesso di disprezzare la «realtà» in cerca di un assoluto irraggiungibile. La guerra aveva, infatti, profanato la nobile dimora di Pico, che aveva assistito alla stesura di tutte le opere della prima stagione dello scrittore. Come risulta dalle citazioni, nel romanzo sono presenti allusioni a fenomeni storicamente accertati, a fatti storici di memoria collettiva, cioè di storia, ovvero di fenomeni più complessi, che coinvolgono anche, e soprattutto, il piano della cultura, dell'ideologia e dell'immaginario: la Resistenza, il colonialismo e le marocchinate. ${ }^{[6]}$

[6] Per quanto riguarda la richiesta dei giusti risarcimenti ai francesi a causa delle marocchinate, il ricorso presso il Tribunale Militare di Roma e presso la Corte internazionale non è ancora chiuso. Emiliano Ciotti, presidente dell'Associazione Vittime delle Marocchinate, fornisce i numeri di questo massacro: «Nella seduta notturna della Camera del 7 aprile 1952 la deputata del PCI Maria Maddalena Rossi denunciò che solo nella provincia di Frosinone vi erano state 60.000 violenze da parte delle truppe del generale Juin». (http://www. lastampa.it/2017/03/16/cultura/la-verit-nascosta-delle-marocchinate-saccheggi-e-stupri-delle-truppecoloniali-francesi-in-ciociaria, pagina consultata il 15/06/2017). 


\section{BIBLIOGRAFIA}

Amigoni, F., 2004, Fantasmi del Novecento, Bollati Boringhieri, Torino.

Bo, C., 1991, Prefazione, in: Landolfi, I. (a cura di), 1991, Tommaso Landolfi, Opere I (1937-1959), Rizzoli, Milano.

Durand G., 2009, Strutture antropologiche dell'immaginario. Introduzione all'archetipologia generale, Dedalo, Bari.

Landolfi, T., 1977, Il tradimento, Rizzoli, Milano.

Landolfi, T., 1968, Le età d'innocenza, in «Corriere della Sera», 29 dicembre 1968.

Landolfi, T., 1954, Ombre, Vallecchi, Firenze.

Landolfi, T., 1991, Quattro casce, in Ombre, ora in LANDOLFI, I. (a cura di) Opere I (1937-1959), Rizzoli, Milano.

Landolfi, T., 1991, Racconto d'autunno, in LANDOLFI, I. (a cura di) Opere I (1937-1959), Rizzoli, Milano.

Landolfi, T., 1998, Rien va, Adelphi, Milano.

Landolfi, T., 1965, Un amore del nostro tempo, in LANDOLFI, I. (a cura di), 1992, Opere II (1960-1971), Rizzoli, Milano.

Sanguineti, E., 2010, Cultura e realtà, Feltrinelli Editore, Milano.

Siciliano, E., 2009, Prefazione a «Des mois» (1991), in CORTELLESSA, A. (a cura di), 2009, Scuole segrete. Il Novecento italiano e Tommaso Landolfi, Nino Aragno Editore, Torino.

Strazza, M. 2010, Senza via di scampo. Gli stupri nelle guerre mondiali, CONSIGLIO REGIONALE DELLA BASILICATA. Commissione Regionale per la Parità e le Pari Opportunità, Potenza.

Verdenelli, M., 1997, Prove di voce: Tommaso Landolfi, Edizioni dell'OrsO, 1997, Alessandria.

Zangrandi, S., 2011, Cose dell'altro mondo. Percorsi nella letteratura fantastica italiana del Novecento, ArchetipoLibri, Bologna. 
Cionci, A., 2017, La verità nascosta delle "marocchinate", saccheggi e stupri delle truppe francesi in mezza Italia, in La Stampa 16/03/2017, https:// www.lastampa.it/2017/03/16/cultura/la-verit-nascosta-delle-marocchinatesaccheggi-e-stupri-delle-truppe-coloniali-francesi-in-ciociariastDjcmY65lqhNlHtQjfyLL/pagina.html, pagina consultata il 12/10/2018 


\section{BETWEEN LITERATURE AND TRUTH: LANDOLFI'S IMAGINATION IN THE SHORT NOVEL RACCONTO D'AUTUNNO}

Tommaso Landolfi (1908-1979), a representative writer of both European and strongly Italian culture, fits into the category of twentieth century fiction, the so-called linguistic fiction. In 1947 he wrote the short novel Autumn Story, which commemorates the destruction of the ancient abbey of Monte Cassino. It used to be the dearest place of the author's childhood, including the old family estate, which he perceived (as he mentions in the novel), as a real desecration. It is a novel full of metaphorical meanings, ranging from war as a metaphor for human cruel indiscretion, to the house itself, as an old aristocratic residence visited by the main characters. In the light of some biographical data, the contribution attempts to analyze this tragic yet mythical dimension of the pain Landolfi experienced in relation with the beloved house, forcibly destroyed by escaping soldiers. The war, in fact, desecrated the ancient aristocratic house in Pico, where almost all the works of the writer's first period were created and which represented for him a certain protective shell. The study primarily focuses on the perception of some war experiences within the Italian culture of the time (the bombing and other war events in the area of Ciociaria in 1943).

Keywords: Italian culture, Landolfi, imagination, house

\section{NA RASPONU KNJIŽEVNOSTI I ISTINE: LANDOLFIJEVO MAŠTANJE U KRATKOM ROMANU RACCONTO D'AUTUNNO}

Tommaso Landolfi (1908. - 1979.), predstavnik europske te istovremeno i talijanske kulture, uklapa se u kategoriju jezične fantastike dvadesetoga stoljeća. Godine 1947. napisao je kratki roman Racconto d'autunno koji evocira uništenje drevnog samostana Monte Cassinija i njegova najdraža mjesta djetinjstva, uključujući staru obiteljsku kuću što je doživio (kako spominje u romanu) kao pravo oskvrnuće. Riječ je o romanu punom metaforičkih značenja, počevši od rata koji je zapravo metafora za okrutnu ljudsku beznačajnost, pa sve do same kuće na koju glavni lik nailazi na svome putu. U svjetlu nekih biografskih podataka, članak analizira tu tragičnu i mitsku dimenziju boli koju Landolfi osjeća prema uništenoj 
kući te devastacije koju ljudi često u svome bijegu nose sa sobom. Rat je oskvrnuo drevnu aristokratsku kuću u Picu, u kojoj je pisac stvorio skoro sva djela iz svoga prvog razdoblja, te je ona za njega predstavljala zaštitnu ljusku. Istraživanje se prvenstveno usredotočuje na percepciju nekih ratnih iskustava (bombaški napad i ostale ratne događaje na području Ciociaria 1943.) unutar talijanske kulture toga doba.

Ključne riječi: talijanska kultura, Landolfi, prikazi, kuća 